Cahiers $d u$ MONDE RUSSE

\section{Cahiers du monde russe}

Russie - Empire russe - Union soviétique et États indépendants

$52 / 4 \mid 2011$

Varia

\title{
Martin Krispin, «Für ein freies Rußland...»
}

\section{Gábor T. Rittersporn}

\section{(2) OpenEdition \\ Journals}

Édition électronique

URL : http://journals.openedition.org/monderusse/7617

DOI : 10.4000/monderusse.7617

ISSN : $1777-5388$

Éditeur

Éditions de l'EHESS

Édition imprimée

Date de publication : 20 décembre 2011

Pagination : 734-739

ISBN : 978-2-7132-2353-2

ISSN : $1252-6576$

\section{Référence électronique}

Gábor T. Rittersporn, « Martin Krispin, «Für ein freies Rußland...»», Cahiers du monde russe [En ligne], 52/4 | 2011, mis en ligne le 30 novembre 2012, Consulté le 25 septembre 2020. URL : http:// journals.openedition.org/monderusse/7617 ; DOI : https://doi.org/10.4000/monderusse.7617

Ce document a été généré automatiquement le 25 septembre 2020.

(c) École des hautes études en sciences sociales 


\title{
Martin Krispin, «Für ein freies Rußland...»
}

\author{
Gábor T. Rittersporn
}

\section{RÉFÉRENCE}

Martin KRISPIN, «Für ein freies Rußland...». Die Bauernaufstände in den

Gouvernements Tambov und Tjumen 1920-1922. Heidelberg : Universitätsverlag Winter, 2010, 493 p.

1 Max Weber croyait en la science objective. Il était convaincu que le chercheur peut éviter toute motivation personnelle et les jugements de valeur. Les travaux sur le soulèvement de Tambov cruellement réprimé par les bolcheviks constituent une preuve de plus contre son optimisme. Les auteurs ne prennent pas nécessairement parti ouvertement. Mais même le lecteur le plus naïf comprend vite d'où ils parlent.

2 Le verdict des historiens soviétiques est évident. Malgré leurs prétentions d'impartialité, le caractère contre-révolutionnaire de la révolte ne faisait aucun doute pour eux. Il fallait l'écraser pour sauver ce qu'ils prenaient pour la révolution, quel que soit le prix de la victoire qu'ils évitaient soigneusement de détailler ${ }^{1}$. D'une manière ou d'une autre, le reste de la confrérie est parti en guerre contre les apologètes soviétiques. Le sous-texte des travaux des uns est la satisfaction de croire précisément une contre-révolution à l'œuvre, alors que les autres célèbrent chez les insurgés d'authentiques révolutionnaires, ou cherchent à montrer qu'ils se battaient pour des valeurs russes, paysannes, démocratiques, socialistes, communistes oppositionnelles, libertaires ou anarcho-communistes ${ }^{2}$.

3 Il serait peut-être exagéré de fêter le livre de Martin Krispin comme un pas de géant vers l'idéal weberien, mais il y a de la nouveauté dans son engagement. Ses sympathies sont claires. Malgré tout, il est toujours prêt à les nuancer et à accepter d'interpréter des textes et gestes des insurgés comme positions qu'il ne partage pas. De plus, même ses observations désobligeantes sur les certitudes bolcheviques s'accompagnent du 
souci d'analyser les documents dans le contexte concret de leurs opérations stratégiques. Krispin milite pour plus que ses convictions politiques. Sans polémiquer avec les idées reçues de ses collègues, il fait tout pour éviter les pièges où ils tombent et tente prudemment de s'en tenir à la proposition de Weber. L'un des fils conducteurs de son travail consiste à insister sur les carences de la littérature, l'autre est une ferme volonté de professionnalisme.

4 La documentation n'a pas facilité sa tâche. Certes, les sources que le chercheur peut exploiter aujourd'hui sont incomparablement plus riches que ce qui était accessible dans le passé. Krispin a dépouillé un nombre impressionnant de fonds dans les archives centrales et régionales. Ce travail de bénédictin lui a permis une approche critique des matériaux qu'on trouve à Amsterdam, à New York et à Stanford. Il en va de même des articles de la presse des années 1920, des Mémoires de participants et de témoins des événements et des travaux savants.

5 Mais la nature des documents pose un énorme problème. La plupart des éléments doivent être distillés de sources qui proviennent des autorités. Le chercheur doit être conscient du fait que la police secrète, les officiels du parti et les militaires étaient loin de rapporter avec exactitude les actions des rebelles, de reproduire fidèlement leurs écrits, leurs projets et leurs paroles, et de fournir des informations fiables sur leurs personnes. Krispin procède avec grande circonspection. Il lui arrive même de douter de la véracité de renseignements qui pourraient pourtant apporter de l'eau à son moulin.

6 Un autre problème de taille consiste en ce que de de nombreuses sources utilisées par l'auteur viennent d'être exploitées par un confrère. En 2008, Eric C. Landis a publié un livre qui porte sur une partie du sujet de Krispin ${ }^{3}$. Mais le premier a passé en revue seulement la révolte de Tambov alors que le second a essayé de la mettre dans une perspective plus large en la comparant à l'insurrection qui a eu lieu au même moment dans la région de Tjumen'.

7 L'auteur dessine le profil de deux régions qui avaient beaucoup en commun. Toutes les deux comptaient des agriculteurs à la tête d'exploitations intégrées dans les échanges de l'économie nationale et parfois même exportateurs de leurs produits vers l'étranger. Toutes les deux avaient été sévèrement touchées par les vicissitudes de la Grande Guerre, toutes les deux avaient bénéficié de la révolution et souffraient plus que les autres provinces de la politique d'approvisionnement forcé des bolcheviks. Ces derniers soupçonnaient les paysans des deux contrées d'être de petits capitalistes hostiles au régime et des accapareurs de grains. Dans une large mesure les préjugés et les politiques des bolcheviks procédaient de leur vision simpliste de la stratification sociale du village et de leur fantasme de luttes de classes qu'ils confondaient avec les tensions au sein du milieu paysan.

8 En revanche, les deux régions se différenciaient grandement par les origines de leur population. Celle de Tambov était établie depuis des siècles. Elle était liée aux cosaques des territoires méridionaux, à tout le moins dans l'imaginaire populaire. Le sort de Tjumen' était étroitement lié à la colonisation de la Sibérie, aux migrations vers l'est, à l'exil et à la présence remarquable d'anciens bannis et sectaires. Une autre différence résidait dans l'occupation blanche de cette partie de la Sibérie pendant la guerre civile et le refus des agriculteurs de soutenir les occupants ce qui n'empêcha pas les rouges de les prendre pour des contre-révolutionnaires.

9 Landis ne brosse pas un panorama socio-économique de la région et ne tente pas d'y replacer le soulèvement. Il suit les diverses étapes du conflit et celles de l'écrasement 
de la révolte et de la pacification de la province essentiellement entre 1920 et 1922 . Son récit est largement chronologique et aisé à lire. Krispin construit son sujet dans une durée plus longue en faisant le tour de divers problèmes qu'il traite dans des chapitres séparés. L'historien fait seulement un survol rapide des événements en tant que tels mais propose d'excellentes analyses du contexte social et culturel des insurrections, de l'univers des rebelles et de leurs visions politiques.

Le contraste entre les espoirs que la révolution a éveillés et l'expérience de la saisie de plus en plus implacable des produits agricoles est évident. Il est moins évident que, pour les paysans, le coup d'État bolchevik a consommé la révolution. Avec les terres qu'ils avaient prises et avec l'accommodement des bolcheviks au fait accompli, ils avaient obtenu à peu près tout ce que le renversement de l'Ancien Régime pouvait leur donner. Krispin attribue trop d'importance à la politique agraire du Gouvernement provisoire et en accorde trop peu à l'occupation et à la distribution de la terre par les paysans eux-mêmes. Mais il voit bien qu'après 1917, les agriculteurs de Tambov et de Tjumen' s'attendaient à ce qu'on les laisse surmonter la crise engendrée par les livraisons obligatoires pendant la Grande Guerre et par l'absence d'une part notable de la main d'œuvre masculine.

11 La collecte impitoyable de la récolte a continué à démanteler leurs exploitations et a fini par les ruiner en 1920 quand leur fut arraché jusqu'aux semences. Il ne restait alors aux paysans de Tambov que dix pour cent des vivres dont ils avaient disposé annuellement avant 1914. Les confiscations arbitraires provoquaient des désordres un peu partout dans le pays. La recherche devrait comparer slogans et gestes des paysans séditieux dans ces désordres et dans les soulèvements de Tambov et Tjumen'. Toujours est-il que si la grosse majorité des accrochages s'arrêtaient aux bords des villages, les groupes armés qui se rassemblèrent autour d'Aleksandr Antonov et tinrent tête aux bolcheviks à Tambov dès 1918 et la résistance armée à Tjumen' ont changé la donne. En principe, ils auraient pu en rester à des combats localisés. Mais entre l'automne 1920 et l'été 1921 à Tambov, et au cours des premiers sept mois de 1921 à Tjumen', les rebelles armés opéraient de concert avec les nouvelles structures administratives et politiques.

12 L'Union de la paysannerie laborieuse à Tambov (Sojuz trudovogo krest'janstva, STK) et les nouveaux soviets de Tjumen' géraient en principe les affaires locales. La première a proposé un programme et créait des organes centraux et locaux, les seconds avaient pour porte-parole l'état-major de leur Armée populaire. Celui-ci diffusait des proclamations, des appels aux soldats de l'Armée rouge et des règlements qui prévoyaient par exemple l'élection et la destitution des officiers. L'Armée populaire de Tjumen' se différenciait notablement des partisans de Tambov par l'absence du culte d'un leader charismatique et une distance par rapport aux chefs.

13 Krispin déçoit les nostalgiques de l'autogestion des masses, de l'anarchisme populaire et de l'attachement des travailleurs aux idées d'Octobre. Il montre qu'il s'agissait de former des administrations au sens propre du terme et que la liberté absolue, la volja, n'était pas à l'ordre du jour. Il montre également que l'une des revendications les plus importantes des insurgés était la convocation d'une Assemblée constituante pour remplacer celle que les bolcheviks avait dissoute en 1918. Si les rebelles ont fêté une révolution, c'était celle de Février 1917. Et s'il voyaient des traitres dans les bolcheviks, ce n'était pas vraiment à cause de leurs promesses non tenues mais parce qu'ils détruisaient les biens et les droits que le village avait gagnés avant Octobre et en partie grâce à Février. 

de Tambov ne se prononçait pas contre le capitalisme. Certes, il ne se prononçait pas pour le capitalisme non plus. Il plaidait pour une économie mixte où la terre et les grandes industries seraient nationalisées, où l'approvisionnement des villes en denrées passerait par des coopératives, où l'État régulerait les prix et les salaires et où la production industrielle serait placée sous le contrôle conjoint des ouvriers et du gouvernement. Krispin pense que la liberté du commerce que voulaient les insurgés signifie leur prédilection pour l'économie du marché. Rien n'est moins sûr. Peu de ruraux pensaient en ces termes. Les éventuels connaisseurs, pour qui marché n'était pas synonyme de bazar, savaient tout aussi bien l'écart que le libre commerce tendait à creuser entre les prix des produits agricoles et ceux des biens dont le village avait besoin. De plus, la fixation des prix par l'État et le contrôle des salaires et de la production par les autorités et les producteurs sont difficilement compatibles avec tout ce qu'on a l'habitude de prendre pour le marché.

Les rebelles condamnaient avec force la violence bolchevique, ils exigeaient l'égalité pour tous (à l'exception des membres de la maison des Romanov), des élections libres, la liberté de parole, de conscience, de réunion, des syndicats, de la presse et de l'éducation ainsi que l'auto-détermination des minorités nationales de Russie. Les bolcheviks ont discrédité l'idée des soviets à Tambov. En revanche, à Tjumen' les rebelles réclamaient tout le pouvoir aux soviets qu'ils voulaient soustraire à la domination des partis.

Krispin est d'accord avec Landis qui ne voit pas les socialistes révolutionnaires en moteur des événements. L'influence de leurs idées et de leurs slogans est indéniable. Certains dirigeants comme Antonov avaient été membres du parti dans le passé et il y avait des socialistes révolutionnaires parmi les insurgés. Mais le parti en tant que tel avait peu de chose à voir avec les deux mouvements.

mérite de Krispin est de dégager le profil du rebelle et de se demander à quel point il était paysan. L'insurgé typique était un père de famille à la tête d'un foyer moyennement prospère. Il n'était ni koulak, ni bandit aventurier comme les bolcheviks l'ont dénoncé. Il n'était pas un contre-révolutionnaire non plus si l'on fait abstraction des hantises bolcheviques. La plupart des mutins avaient milité déjà en 1917 sans néanmoins adhérer à un parti quelconque. Pendant les soulèvements ils étaient secondés par ce qu'on peut appeler, faute de mieux, l'intelligentsia rurale : un certain nombre d'instituteurs, d'agronomes et de médecins. Il n'est pas simple de faire la part de leur contribution aux déclarations politiques ou aux bulletins d'information. L'activité des paysans dans les années 1917 et 1918 indique qu'ils n'avaient pas nécessairement besoin de guides spirituels. Mais il serait peut-être utile d'étudier la question en profondeur.

18 Contrairement à ce que la littérature suggère, l'horizon des rebelles dépassait leur environnement immédiat. Ils cherchaient avec succès des alliés dans les villes. Ils avaient des informations sur les soulèvements dans d'autres régions et sur l'insurrection de Kronstadt. Les espoirs que ces épisodes ont éveillés indiquent une certaine largeur de vue. Il en va de même des rumeurs qui couraient sur les possibilités de joindre force avec d'autres rebellions qui n'existaient pas nécessairement dans la nature. Les révoltés de Tambov avaient l'illusion d'un large mouvement de cosaques, leurs camarades lointains celle d'une Grande Sibérie insurgée.

Cahiers du monde russe, 52/4 | 2011 

l'antisémitisme des rebelles. Il ne cherche pas assidument l'influence de traditions séculaires, il est attentif à tout ce que la culture rurale de l'époque empruntait à d'autres sphères. Il reconnaît ce que les paysans devaient à l'agitprop des socialistes révolutionnaires. Il voit également que les rebelles étaient sans doute plus soucieux de la liberté de conscience que de l'Église et il pense que la religion jouait un rôle plutôt marginal. On pourrait peut-être nuancer sa position et méditer si des attentes millénaristes ne constituaient pas le sous-texte de certains discours et pratiques. Mais il a incontestablement raison quand il conclut que l'antisémitisme véhément de nombre d'insurgés n'avait pas ses origines dans une fixation populaire coutumière. Le phénomène relevait avant tout de la présence de commissaires d'origine juive parmi les cadres de la police politique qui dirigeaient les campagnes d'approvisionnement et la chasse aux rebelles et de l'identification des bolcheviks comme étrangers au monde du village.

Landis prête grande importance à l'introduction de la nouvelle politique économique en mars 1921 : elle aurait notablement aidé à la pacification de Tambov. Krispin est plus prudent. D'une part, c'était le moment où les opérations militaires contre les soulèvements s'intensifiaient à l'extrême. D'autre part, le changement de cap de la politique bolchevique n'empêcha ni la radicalisation du mouvement à Tjumen' ni les activités de l'Union de Tambov. Krispin cite des exemples qui montrent à quel point les paysans se méfiaient des promesses du régime.

21 Il y a une circonstance qui milite contre l'opinion de Landis pour qui l'échec des rebelles signale la fin d'une époque. Il a sans doute raison en ce qui concerne les soulèvements à grande échelle car la résistance des paysans à la collectivisation n'embrassait pas des régions comparables à celle de Tambov et de Tjumen'. Malgré tout, certaines idées qui motivaient les insurgés de ces deux provinces continuèrent leur œuvre, en partie parce qu'elles avaient surgi bien avant 1920. L'Union de la paysannerie laborieuse et les soviets de Tjumen' avaient des prédécesseurs : les Unions paysannes (Krest'janskie sojuzy) de la révolution de 1905. Dès 1917, les villageois et les socialistes révolutionnaires tentèrent de les remettre sur pied par endroit. Malheureusement, Krispin ne prête pas attention à ces unions dont l'histoire dans la longue durée mériterait des recherches.

Pourtant, les Unions paysannes avaient la vie dure dans la mesure où elles firent partie du discours contestataire du village jusqu'à la fin des années 1930 environ. Mouvement national pour les uns, syndicat pour les autres, structure de gouvernement pour les troisièmes, les Unions faisaient surface dans les conversations et elles étaient régulièrement rapportées par la police et les organes du parti. S'il n'est pas certain que les rapports disent vrai, ils indiquent au moins la persistance du souvenir des Unions dans la mémoire rurale et l'obsession des autorités. Fait intéressant : le Comité central constatait, à tort ou à raison, que l'agitation pour les Unions paysannes prit une ampleur particulière précisément pendant la crise de la nouvelle politique économique. Selon la police, elles étaient souvent évoquées pendant la campagne des élections au Soviet suprême de 1937.

23 Un autre problème qu'on pourrait explorer dans la longue durée est la nature de la vision politique et des programmes des insurgés. Dans diverses formulations, les éléments en apparaissent tout au long des années 1920 et 1930 dans des tracts et proclamations distribués secrètement ainsi que dans des projets de petit groupes 
clandestins de protestataires qui n'étaient pas les fruits de l'imagination de la police politique. Les réfugiés soviétiques interrogés en Occident après la guerre partageaient ces idées aussi bien que nombre de participants des courants dissidents des années 1960-1980 et même des réformateurs qui s'agitaient à la veille de la chute du régime soviétique.

Krispin a fait un formidable travail de recherche. Le malheur veut que sa manière d'exposer les résultats ne soit pas tout à fait à la hauteur. Une construction plus soigneuse du livre aurait pu éviter des obscurités, des redites et l'épreuve du lecteur qui doit parfois rechercher l'endroit où tel ou tel autre fait répété est expliqué en détail. L'éditeur n'a pas cru bon de faciliter ces labeurs en attachant un index.

Les bolcheviks et leurs historiens ont sévi contre les rebelles au nom d'un progrès que leur régime était censé couronner. Leurs adversaires les imitent dans la mesure où ils se réclament de leur propre telos: utopies sympathiques et moins sympathiques et diverses versions des meilleurs des mondes possibles. Grâce à son souci de professionnalisme Krispin parvient, plus que ses collègues, à éloigner l'histoire des idées reçues.

\section{NOTES}

1. I.P. Donkov, « Organizacija razgroma antonovščiny », Voprosy istorii KPSS, n 6, 1966, p. 59-71; id.; Antonovščina: zamysl' i deistvitel'nost', M., 1977; N.F. Bugai, Črezvyčainye organy sovetskoj vlasti : Revkomy 1917-1921, M., 1980; D.L. Golinkov, Kruščenie antisovetskogo podpol'ia v SSSR, M., 1972; I.Ia. Trifonov, "Iz istorii razgroma antonovščiny », Voenno-istoričeskij žurnal, n 9, 1964, p. 27-50; B. Roitman, « Rol' kavbrigady G.I. Kotovskogo v razgrome antonovščiny », ibid., n 6, 1981, p. 71-74; K.P. Orlov, « K istorii likvidacii antonovskogo matježa (1921 g.) », Istoričeskij Arhiv, n 4, 1962, p. 203-208.

2. S.A. Esikov, V.V. Kaniščev, “ “Antonovskij NEP”. Organizacija i dejatel'nost' “Sojuza trudovogo krest'janstva" Tambovskoj gubernii 1920-1921 », Otečestvennaja istorija, n 4, 1993, p. 60-72 ; V.V. Samoškin, Antonovskoe vosstanie, M., 2005; N.V. Fatueva, Protivosostojanie: Krizis vlasti, tragedija naroda. Iz istorii krest'janskih volnenii i vosstanii v Tambovskoj gubernii v 1918-192 gg., Riazan', 1996 ; Oliver H. Radkey, The Unknown Civil War in Russia: A Study of the Green Movement in the Tambov Region, Stanford, 1976; Aleksandr Skirda, Les Anarchistes dans la révolution russe, P., 1973; id., Les Anarchistes russes, les soviets et l'autogestion, P., 1973; Vladimir Brovkin, Behind the Front Lines of the Civil War: Political Parties and Social Movements in Russia, 1918-1922, Princeton, 1994; Andrea Graziosi, The Great Soviet Peasant War: Bolsheviks and Peasants 1917-1933, Cambridge, MA, 1996.

3. Eric C. Landis, Bandits and Partisans: The Antonov Movement in the Russian Civil War, Pittsburgh : University of Pittsburgh Press. Alexandre Sumpf a rendu compte de l'ouvrage dans les Cahiers du Monde russe, 49/4 (2008), p.736-738. 